\title{
A Single Dose TMV-HA Vaccine Protects Mice from H5N1 Influenza Challenge
}

\author{
Jyothi K Mallajosyula ${ }^{1}$, Trushar Jeevan ${ }^{2}$, Rachel Chikwamba ${ }^{3}$, Richard J Webby ${ }^{2}$, Alison A McCormick ${ }^{1 *}$ \\ ${ }^{1}$ Touro University California, Vallejo CA, USA \\ ${ }^{2}$ St. Jude Children's Research Hospital, Memphis TN, USA \\ ${ }^{3}$ Council for Scientific and Industrial Research (CSIR), South Africa
}

Received: January 22, 2016; Accepted: January 27, 2016; Published: February 25, 2016

*Corresponding author: Alison A. McCormick, College of Pharmacy, Touro University, California, Tel: +707-638-5987; Fax: +707-638-5959; E-mail: alison.mccormick@tu.edu

\begin{abstract}
Recombinant subunit vaccines are an efficient strategy to meet the demands of a possible influenza pandemic, because of rapid and scalable production. However, vaccines made from recombinant Hemagglutinin (HA) subunit protein are often of low potency, requiring repeated boosting to generate a sustained immune response. Previously, we demonstrated improved immunogenicity of a plant-made H1 Hemagglutinin (HA) vaccine by chemical conjugation to the surface of the Tobacco Mosaic Virus (TMV) which is non infectious in mammals. Antigen coated TMV is taken up by mammalian dendritic cells and is a highly effective antigen carrier for subunit protein vaccines. In this work, we tested the effectiveness of a TMV-H5 HA conjugate vaccine. We compared the TMV-H5 immunogenicity in mice, with and without an oil-in water squalene adjuvant, to H5N1 virus or HA protein alone, as measured by anti-H5 IgG titers and Hemagglutination Inhibition (HAI). We then evaluated the efficacy of the TMV-H5 vaccine by lethal H5N1 virus challenge in mice. Our results show that a single dose of the TMV-H5 conjugate vaccine is sufficient to generate $40 \%$ survival, similar to H5 protein given with adjuvant, or $100 \%$ survival after vaccination with adjuvant, similar to H5N1 virus vaccination.
\end{abstract}

Keywords: Influenza; H5N1; Nanoparticle; Plant made pharmaceuticals; Tobacco Mosaic Virus (TMV); Hemagglutinin (HA); Subunit protein; Vaccination; Dose sparing

\section{Abbreviations}

Tobacco Mosaic Virus (TMV); Hemagglutinin (HA); H5N1 HA (H5); Enzyme linked Immunosorbent Assay (ELISA); Bicinchoninic Acid Assay (BCA); Hemagglutinin Inhibition (HAI); Phosphate Buffered Saline (PBS); MF-59-like Squalene Adjuvant AddaVax ${ }^{\mathrm{TM}}$ (Sq); 1-Ethyl-3-(3-dimethylaminopropyl) carbodiimide/N-hydroxysuccinimide (EDC/NHS)

\section{Introduction}

Vaccination against influenza A infection has undoubtedly saved millions of lives, but zoonotic influenza represents a continuous source of potentially new infectious virus strains, either by mutation to promote human infections, or by genetic shift through reassortment with a human seasonal influenza.
Since 1997, H5N1 has emerged as a potentially pandemic virus from human contact with infectious birds [1], with an alarmingly high fatality rate of $\sim 53 \%$ [2]. Unfortunately the H5N1 virus continues to generate a high rate of mutation, and many new clades of virus [3-5]. This is of special concern, because 1) the strain banked against a possible pandemic may not be immunologically relevant [6], 2) the poor efficacy of the current vaccine will require two immunizations (with adjuvant) to achieve homologous or heterologous immunity in vaccinated individuals [7, 8], or 3) that it will take months to scale up embryonated egg-based vaccine production, which may be too slow to facilitate mass immunization.

Significant progress has been made to rapidly produce H5N1 vaccines by expression of the Hemagglutinin (HA) protein as the vaccine agent. HA protein is the main stimulus of virus neutralization, and can be made independently of the whole virus. Production is much safer, simpler and faster [9], and H5 HA immunization generates protective immunity in mice [10], ferrets $[11,12]$ and in humans [13-15]. But, H5 HA protein is weakly immunogenic, and requires boosting and/or adjuvant use for inducing effective immunity, so immune potency is still an issue. We have optimized HA formulation by chemically associating $\mathrm{H} 5$ HA protein to the surface of, Tobacco Mosaic Virus (TMV), a noninfectious plant virus. TMV is an attractive virus carrier, because it has the optimal size to promote immune cell uptake $[16,17]$, can be made at Kg scale in planta with a minimum of processing steps, and is not a mammalian pathogen [18]. We have developed a TMV coat protein variant that expresses a surface exposed lysine [19] that allows for high density association of HA protein to the virus surface, using a one step conjugation chemistry [20]. In this study, we have greatly improved the low immunogenicity of H5 HA subunit vaccination by TMV-H5 conjugation, with equivalent immune activation potency as an MF-59-like squalene adjuvant. This could be an important advance in development of dose-sparing protective HA vaccines that are based on subunit antigen delivery by virus co-formulation. 


\section{Materials and methods}

\section{Conjugation and Vaccine preparation}

Chemical conjugation of an H5 HA1 Fc fusion protein (10003V06H1, Sino Biological Inc.,) to the surface of lysine modified TMV virus [19], was done as previously described [20]. Conjugation reactions were considered complete when no free HA protein was visible by SDS-PAGE. After conjugation, reactive agents were removed by overnight dialysis against PBS in Slide-a-Lyzer cartridge (Thermo-Scientific), and the protein quantity was determined by the Bicinchoninic Acid Assay (BCA;BioRad), and HA content was quantified by Enzyme Linked Immunosorbent Assay (ELISA), to normalize the vaccine dose.

\section{Vaccination and immune response evaluation in mice}

BALB/c mice (Charles River, CA) were housed at Touro University according to guidelines established in the Care and Use of Animals and according to IACUC approved protocols. Mice were given a $100-200 \mu \mathrm{l}$ subcutaneous (s.c.) injection of $7.5 \mu \mathrm{g}$ HA protein (unconjugated), or the equivalent $\mathrm{HA}$ as a $15 \mu \mathrm{g}$ conjugate, with or without a squalene based oil-in-water emulsion adjuvant AddaVax ${ }^{\mathrm{TM}}$ (1:1 ratio; InvivoGen). Inactivated H5N1 virus was used as a positive control, and pre-immune serum was used to determine baseline sera titers. Vaccines were administered on day one and day 30 , and sera was collected by tail vein bleed on days 14 and 28 days post vaccine $1(\mathrm{pV} 1)$ or day 44, 14 days post vaccine 2 (pV2) for ELISA analysis.

ELISA was used to determine serum IgG titers as previously described [21]. Anti-H5 titers reported in $\mathrm{ng} / \mathrm{ml}$ were determined from a standard curve generated by a 3-fold serial dilution of a 10 $\mathrm{ng} / \mathrm{ml}$ mouse anti-HA monoclonal antibody (DPJY01, BEI \#NR19869). Hemagglutinin Inhibition (HAI) titers of the sera were then determined by the standard microtitration method [22], with serial 2 -fold dilution of serum mixed with $0.5 \%$ turkey Red Blood Cells (RBCs; Innovative Research, Inc.) in sterile phosphate buffered saline (PBS;Gibco).

\section{H5N1 virus challenge}

H5N1 virus challenge studies were conducted under animal Bio-safety level 3 (ABSL3) conditions and according to St. Jude Children's Research Hospital approved institutional procedures and protocols. Ten week old BALB/c mice were vaccinated once, using $15 \mu \mathrm{g}$ H5 protein equivalent, or two times 14 days apart with $7.5 \mu \mathrm{g}$ H5 protein equivalent, with or without squalene based adjuvant. At day 26, serum was collected for ELISA and HAI analysis, and on day 28, mice were challenged intranasally with 10xLD50 of highly pathogenic avian A/Vietnam/1203/04, diluted in $50 \mu \mathrm{L}$ of PBS, as previously described [23]. Mice were monitored daily for $>25 \%$ weight loss, neurologic signs, or motor dysfunction, and were euthanized according to IACUC approved procedures.

\section{Results and discussion}

\section{TMV-H5 HA conjugation and pilot immunization}

As shown in Figure 1, we used EDC/NHS (1-Ethyl-3-(3-

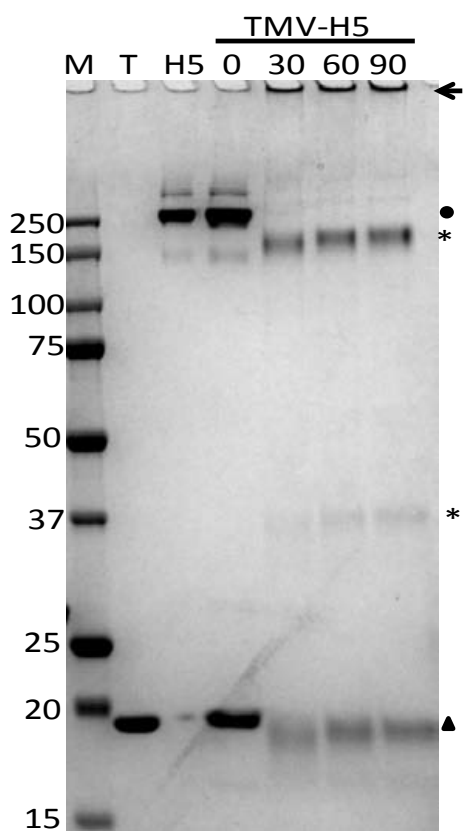

Figure 1: Preparation of the TMV-H5 conjugate vaccine.

A 1:1 mass ratio of TMV-lysine (T: filled triangle) and H5 HA1 protein (H5: filled circle; Sino Biologicals \#10003-V06H1; homologous to A/ Vietnam/1203/2004) were reacted in the presence of EDC/NHS for $0,30,60$ or 90 minutes, and then stopped with the addition of $10 \mathrm{mM}$ methylamine. Reactions were dialyzed against PBS, and then $3 \mu \mathrm{g}$ protein was separated by an $8-16 \%$ gradient SDS-PAGE (BioRad), compared to Precision Plus molecular weight standard (BioRad). High molecular weight conjugate product (arrow) and TMV dimers/ mulitmers (asterisks) are seen after 30 minutes, and are not increased by further incubation time.

dimethylaminopropyl) carbodiimide/N-hydroxysuccinimide) conjugation of H5 HA (H5 HA1-Fc protein; Sino Biological Inc.) to a lysine modified TMV as previously described [20]. After 30 minutes, both TMV (triangle) and HA (circle) are reduced, and a high molecular weight aggregate (TMV-H5) accumulates in the gel above $250 \mathrm{kDa}$, and in the stack after 8-16\% SDS-PAGE separation (arrow). A TMV dimer and tetramer are also visible (asterisks).

To assess immune response titers, $7.5 \mu \mathrm{g}$ of HA equivalent protein as TMV-H5 conjugate was used to immunize mice $(n=3)$, with or without a squalene based oil-in-water adjuvant (Sq; InvivoGen). Inactivated $\mathrm{H} 5 \mathrm{~N} 1$ virus was also used as a positive control, and H5 protein given +/-Sq was used to control for HA immune reactivity without TMV. Mice were tested for anti-HA IgG 14 and 28 days after a single dose, and at day 44, 14 days after a boost on day 30. As shown in Figure 2A, all immunized mice had measureable anti-H5 specific IgG titers after a single dose of TMV-H5, when compared to an anti-H5 monoclonal antibody standard (DPJY01, BEI \#NR-19869). Only TMV-H5 with Sq had significantly better immune titers $(* \mathrm{p}<0.05)$ than any of the other vaccine groups, suggesting synergy in H5 immune potentiation between TMV and adjuvant. Notably, TMV-H5 had statistically similar immune titers compared to H5 HA 

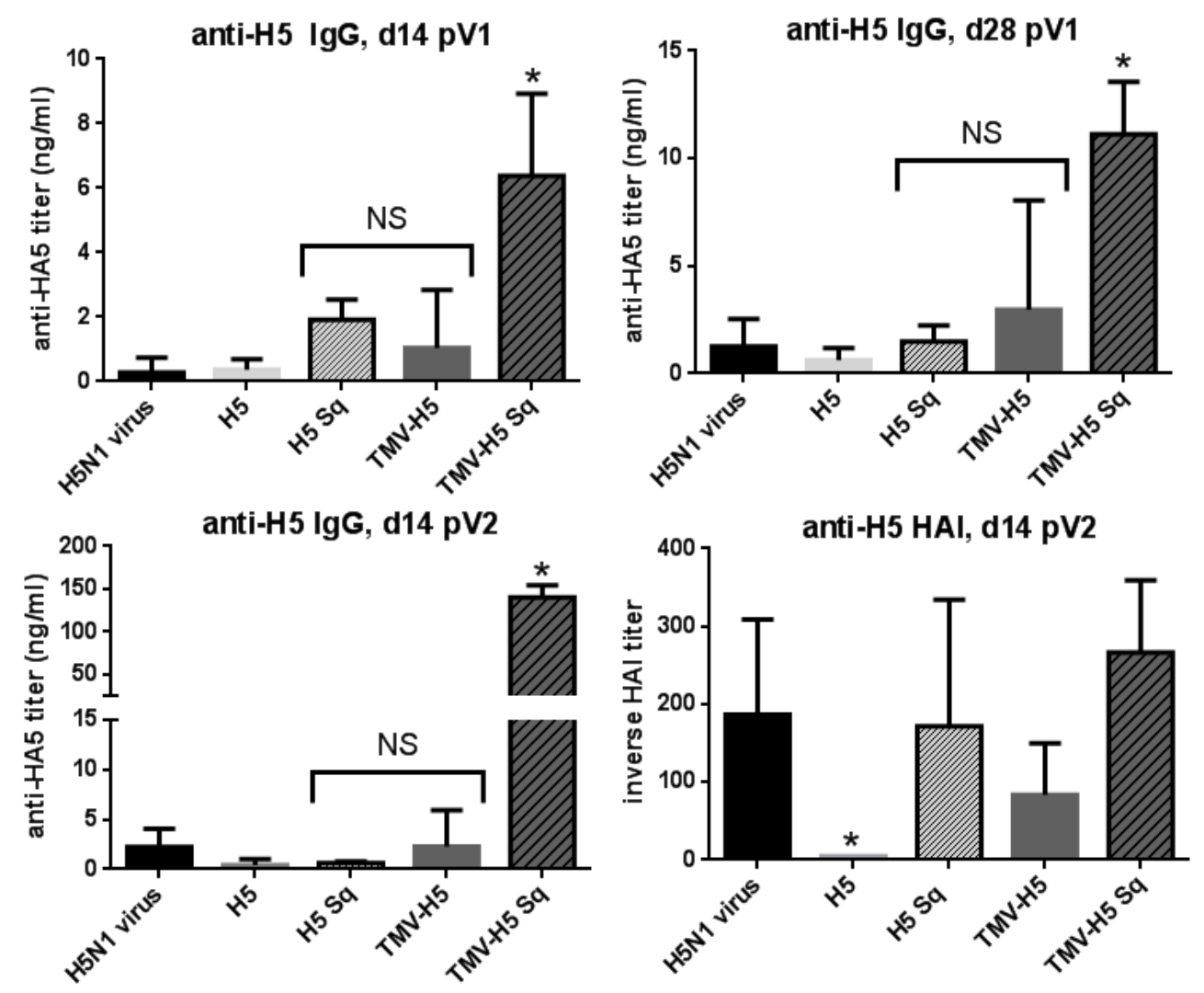

Figure 2: TMV-H5 vaccine pilot study; IgG titers and HAI. Mice were immunized one time with $7.5 \mu \mathrm{g}$ of H5 equivalent protein, or H5N1 virus as a control. At 14 (b1) or 28 (b2) days post vaccine 1 (A/B), serum was collected, and tested for total IgG by ELISA on plates coated with 5 ug/ml baculovirus expressed H5 protein (BEI \# NR-10510). Serum responses were quantified against a standard curve of 10ng/ml anti-H5 IgG (DPJY01, BEI \#NR-19869), and differences in response are indicated (significant $*$ : $\leq 0.05$;NS:not significantly different). One additional dose was administered at day 30, and bleed 3 serum responses were measured at day 44 by the same method (C). Relevant H5N1 virus (A/Vietnam/1203/2004) was titered for turkey RBC agglutination (D). Virus at 4 fold greater than the minimum agglutinating titer was then pretreated with serial dilutions of RDE-treated serum from study sera. After 1 hour, $0.05 \%$ turkey red blood cells were added, and HAI titer was measured as the inverse of the minimum dilution blocking the formation of RBC agglutination (RBC buttons). TMV vaccination gave titers equivalent to pre-immune sera (not shown).

protein given with squalene (NS; not significantly different), indicating that TMV provides adjuvant activity equivalent to that of squalene emulsions [20]. After two weeks (Figure 2B), the immune response to TMV-H5 Sq nearly doubled (mean 6.3 vs 11.1), suggesting that TMV-HA vaccines may also provide a depot effect. Two weeks after a second immunization (Figure 2C), TMV-H5 Sq boosted mice had a log increase in IgG titer (mean 11.1 vs 140.0 ) which was significantly better than other vaccine groups $(\mathrm{p}<0.001)$, and all other immune responses were statistically similar to each other (NS).

Although IgG titers were low for both H5N1 virus and H5 HA Sq after two immunizations, significant HAI titers $(>40)$ were measured for all vaccine groups except HA protein given without adjuvant $(* p<0.05$; Figure $2 D)$, indicating both quantitative and qualitative boosting of TMV-HA conjugate vaccines.

\section{Immunization and protection from H5N1 viral challenge}

As shown in Figure 3A, mice were immunized following a similar strategy in the pilot study. Mice $(n=10)$ were vaccinated with either one dose (15 $\mu$ g total HA protein) on day 1 , or two doses on days 1 and 14 (7.5 $\mu$ g each dose, $15 \mu$ g total HA protein), with or without oil-in-water squalene (Sq) adjuvant as indicated. HA only was not tested in this study due to lack of IgG and HAI responses in the pilot study. Sera were collected on day 28, and mice were challenged on day 30 by intranasal administration of 10xLD50 of A/Vietnam/1203/04. Mice were monitored for symptoms of infection (weight loss, neurological or behavioral changes), and euthanized according to IACUC protocols. As shown in Figure 3B, IgG immune response titers were slightly different than the pilot, especially in regards to inactivated H5N1 virus responses, which were much higher than in the pilot, possibly due to differences in preparations and estimates of HA content. Another difference, was the low IgG induced by TMV-HA, after either one or two vaccines. However, after one dose TMV-HA+Sq induced significantly higher IgG titers than $\mathrm{HA}+\mathrm{Sq}(\mathrm{p}<0.05)$ and was not different than inactivated virus immunization. After two doses, TMV-HA+Sq, HA+Sq and H5N1 virus immunization induced similar levels of IgG. In contrast, HAI titers (Figure 3C) for TMV-HA were significantly better after two doses than $\mathrm{HA}+\mathrm{Sq}$, with or without squalene adjuvant addition. 


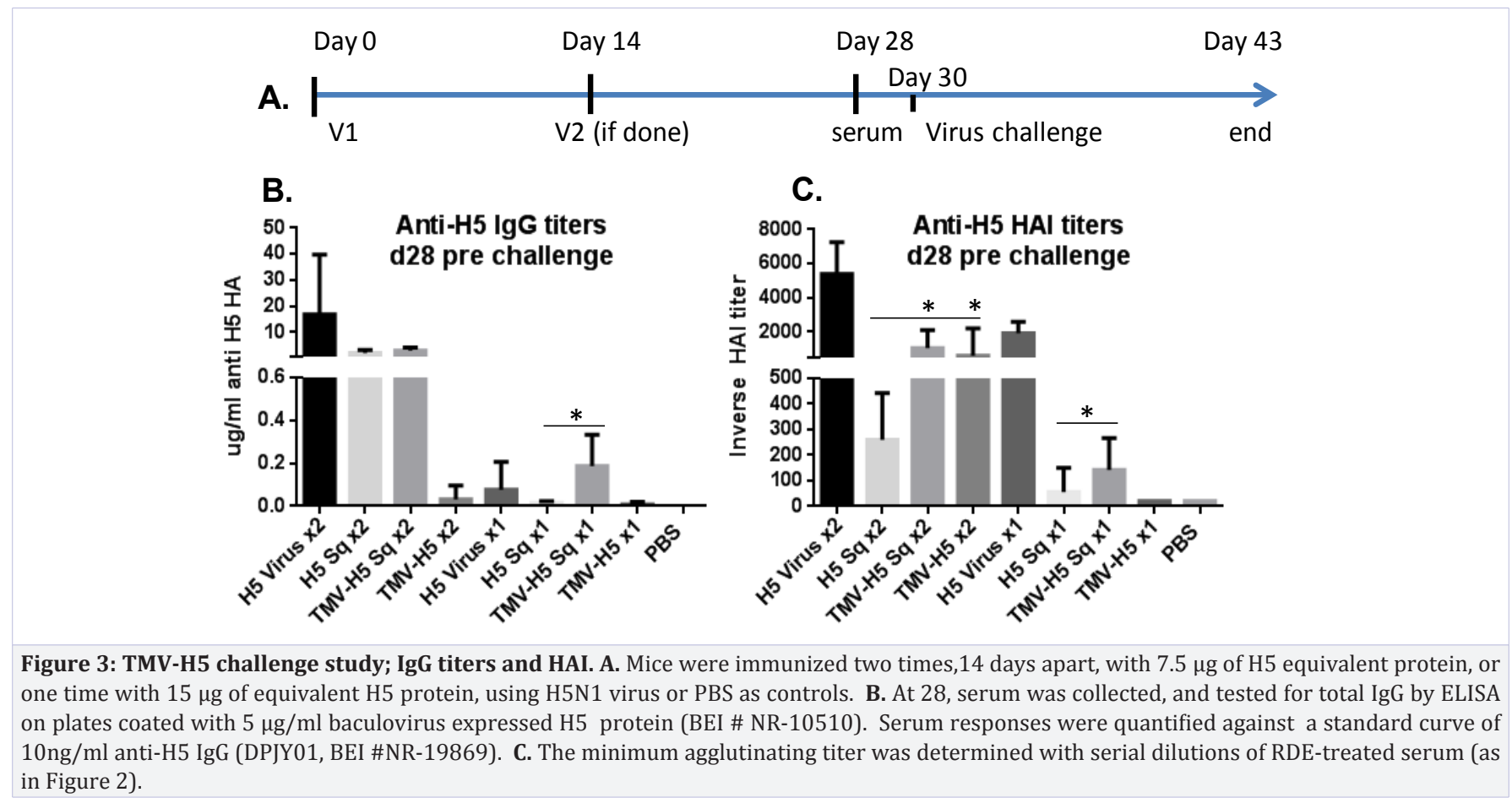

in Figure 2).
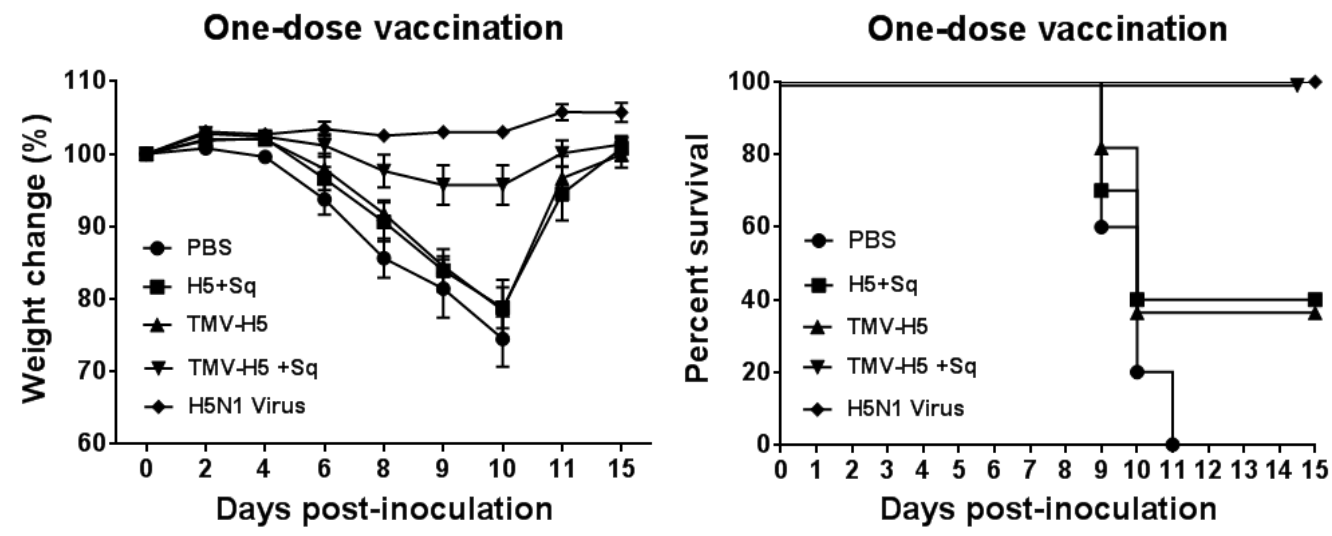

Two-dose vaccination
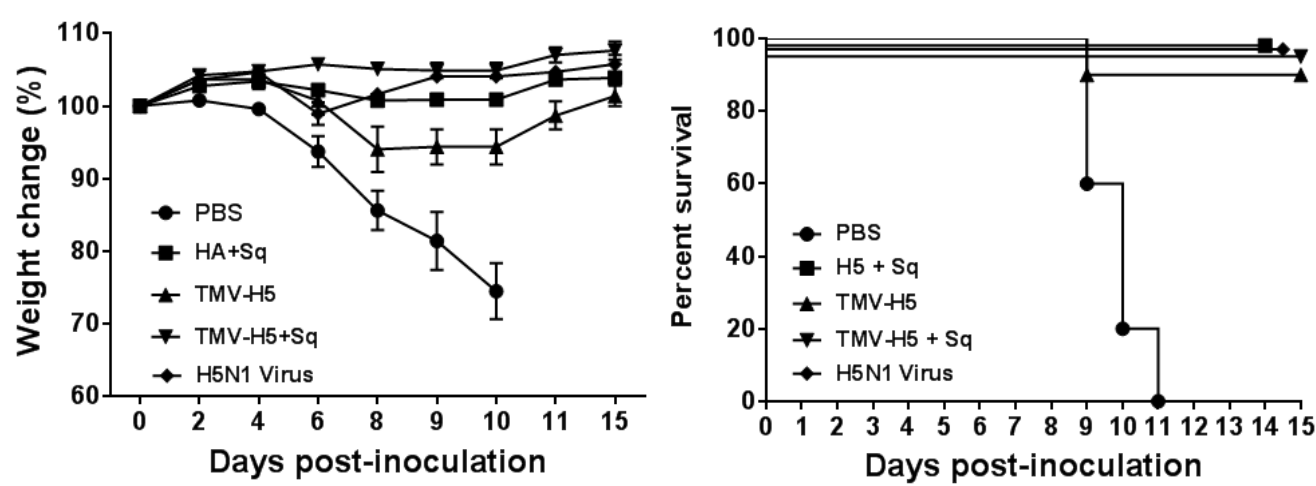

Figure 4: TMV-H5 challenge study; weight loss and survival. Mice were immunized two times, 14 days apart, with 7.5 $\mu$ g of H5 equivalent protein, or one time with $15 \mu \mathrm{g}$ of equivalent H5 protein, using H5N1 virus or PBS as controls. At day 30, mice were challenged by intranasal delivery of 10xLD50 H5N1 virus. Mice were weighed every other day, and euthanized if less than $80 \%$ of starting weight, or had signs of neurological or behavioral distress. A/C. Weights were plotted as the average +/- the standard error of measurement. B/D. Survival was calculated by Kaplan-Meier analysis. 
After a single dose, HAI titer was also significantly better in the TMV-HA+Sq than $\mathrm{HA}+\mathrm{Sq}$ vaccine group $(\mathrm{p}<0.05)$, but was not above background $(<40)$ in the TMV-HA group.

At day 30, mice were challenged with a lethal dose of A/ Vietnam/1203/04, and monitored for weight changes and survival. After one immunization, TMV-HA+Sq generated equivalent protection to inactivated $\mathrm{H} 5 \mathrm{~N} 1$ virus vaccination $(100 \%$ of mice) both in terms of weight loss (Figure $4 \mathrm{~A})$ and survival (Figure 4B). Surprisingly, despite low IgG and low HAI for TMV-HA after one dose (no adjuvant), we measured 40\% survival, equivalent to $\mathrm{HA}+\mathrm{Sq}$ (Figure $4 \mathrm{~B}$ ), indicating that TMV provides immune activation activity similar or equal to that of a squalene-based MF-59-like adjuvant. After two immunizations, all vaccine groups had statistically similar survival (90-100\%; $\mathrm{p}>0.9$ ), irrespective of adjuvant use, and compared to the PBS control $(\mathrm{p}<0.01)$.

Ongoing studies to further improve TMV-HA formulations include intranasal administration, to improve mucosal immunity, multivalent combinations of various TMV-HAs, and additional adjuvant co-formulations. Preliminary data suggest higher mean titers of anti-H5 IgG are achieved with TMV-H5 intranasal dosing, but HAI, microneutralization analysis, cross protection, and challenge studies will be needed to confirm relevance to protection, similar to other studies using HA virus-like particles [24], nanoparticles [25] or virus vaccines [26].

\section{Acknowledgements}

This work was supported by a grant from CSIR.

\section{References}

1. Gambotto A, Barratt-Boyes SM, de Jong MD, Neumann G, Kawaoka Y Human infection with highly pathogenic h5n1 influenza virus. Lancet. 2008;371(9622):1464-1475. doi: 10.1016/S0140-6736(08)60627-3

2. WHO. Influenza monthly risk assessment summary, human infection with avian influenza a(h5) viruses.

3. Vijaykrishna D, Bahl J, Riley S, Duan L, Zhang JX, Chen H, et al. Evolutionary dynamics and emergence of panzootic h5n1 influenza viruses. PLoS pathogens. 2008;4(9):e1000161.

4. Sonnberg S, Webby RJ, Webster RG. Natural history of highly pathogenic avian influenza h5n1. Virus research. 2013;178(1):63-77.

5. Xu L, Bao L, Yuan J, Li F, Lv Q, Deng W, et al. et al. Antigenicity and transmissibility of a novel clade 2.3.2.1 avian influenza h5n1 virus. J Gen Virol. 2013;94 (Pt 12):2616-2626.

6. WHO. Antigenic and genetic characteristics of zoonotic influenza viruses and development of candidate vaccine viruses for pandemic preparedness. 2015;1-10

7. Carter NJ, Plosker GL. Prepandemic influenza vaccine h5n1 (split virion, inactivated, adjuvanted) [prepandrix]: A review of its use as an active immunization against influenza a subtype h5n1 virus. BioDrugs: clinical immunotherapeutics, biopharmaceuticals and gene therapy. 2008;22(5):279-292.

8. Krammer F, Hai R, Yondola M, Tan GS, Leyva-Grado VH, Ryder AB, et al. Assessment of influenza virus hemagglutinin stalk-based immunity in ferrets. Journal of virology. 2014;88(6):3432-3442. doi: 10.1128/ JVI.03004-13. Epub 2014 Jan 8.

9. Skowronski DM, Janjua NZ, De Serres G, Sabaiduc S, Eshaghi A, Dickinson JA, et al. Low 2012-13 influenza vaccine effectiveness associated with mutation in the egg-adapted h3n2 vaccine strain not antigenic drift in circulating viruses. PloS one. 2014;9(3):e92153. doi: 10.1371/journal.pone.0092153. eCollection 2014.

10. Major D, Chichester JA, Pathirana RD, Guilfoyle K, Shoji Y, Guzman CA, et al. Intranasal vaccination with a plant-derived $\mathrm{h} 5$ ha vaccine protects mice and ferrets against highly pathogenic avian influenza virus challenge. Human vaccines \& immunotherapeutics. 2015;11(5):12351243. doi: $10.4161 / 21645515.2014 .988554$.

11. Shoji Y, Bi H, Musiychuk K, Rhee A, Horsey A, Roy G, Green B, et al. Plant-derived hemagglutinin protects ferrets against challenge infection with the a/indonesia/05/05 strain of avian influenza. Vaccine 2009;27(7):1087-1092. doi: 10.1016/j.vaccine.2008.11.108. Epub 2008 Dec 25.

12. Khurana S, Verma S, Verma N, Crevar CJ, Carter DM, Manischewitz J, et al. Bacterial ha1 vaccine against pandemic h5n1 influenza virus: Evidence of oligomerization, hemagglutination, and cross-protective immunity in ferrets. Journal of virology 2011; 85(3):1246-1256. doi: 10.1128/JVI.02107-10. Epub 2010 Nov 17.

13. Ledgerwood JE, Hu Z, Gordon IJ, Yamshchikov G, Enama ME, Plummer $\mathrm{S}$, et al. Influenza virus h5 DNA vaccination is immunogenic by intramuscular and intradermal routes in humans. Clinical and vaccine immunology. 2012;19(11):1792-1797. doi: 10.1128/CVI.05663-11. Epub 2012 Sep 5.

14. Treanor JJ, Essink B, Hull S, Reed S, Izikson R, Patriarca P, et al. Evaluation of safety and immunogenicity of recombinant influenza hemagglutinin (h5/indonesia/05/2005) formulated with and without a stable oil-in-water emulsion containing glucopyranosyl-lipid a (se+gla) adjuvant. Vaccine. 2013;31(48):5760-5765. doi: 10.1016/j. vaccine.2013.08.064. Epub 2013 Sep 27.

15. Cummings JF, Guerrero ML, Moon JE, Waterman P, Nielsen RK, Jefferson S, et al. Safety and immunogenicity of a plant-produced recombinant monomer hemagglutinin-based influenza vaccine derived from influenza a (h1n1)pdm09 virus: A phase 1 doseescalation study in healthy adults. Vaccine. 2014;32(19):2251-2259. doi: 10.1016/j.vaccine.2013.10.017. Epub 2013 Oct 12.

16. Kemnade JO, Seethammagari M, Collinson-Pautz M, Kaur H, Spencer DM, McCormick AA. Tobacco mosaic virus efficiently targets dc uptake, activation and antigen-specific $\mathrm{t}$ cell responses in vivo. Vaccine. 2014;32( 33):4228-4233.

17. McCormick AA, Corbo TA, Wykoff-Clary S, Nguyen LV, Smith ML, Palmer KE, et al. Tmv-peptide fusion vaccines induce cell-mediated immune responses and tumor protection in two murine models. Vaccine. 2006;24(40-41):6414-6423.

18. Liu R, Vaishnav RA, Roberts AM, Friedland RP. Humans have antibodies against a plant virus: Evidence from tobacco mosaic virus. PloS one. 2013;8(4):e60621. doi: 10.1371/journal.pone.0060621. Epub 2013 Apr 3.

19. Smith ML, Lindbo JA, Dillard-Telm S, Brosio PM, Lasnik AB, McCormick AA, et al. Modified tobacco mosaic virus particles as scaffolds for display of protein antigens for vaccine applications. Virology. 2006;348(2):475-488.

20. Mallajosyula JK, Hiatt E, Hume S, Johnson A, Jeevan T, Chikwamba R, et al. Single-dose monomeric ha subunit vaccine generates full protection 
from influenza challenge. Human vaccines \& immunotherapeutics. 2013;10(3):586-595.

21. Mc Cormick AA, Kumagai MH, Hanley K, Turpen TH, Hakim I, Grill $\mathrm{LK}$, et al. Rapid production of specific vaccines for lymphoma by expression of the tumor-derived single-chain $\mathrm{fv}$ epitopes in tobacco plants. Proceedings of the National Academy of Sciences of the United States of America. 1999;96(2):703-708.

22.WHO. Prevention and control of influenza with vaccines: Recommendations of the advisory committee on immunization practices (acip), 2011. American journal of transplantation: official journal of the American Society of Transplantation and the American Society of Transplant Surgeons. 2011;11(10):2250-2255. DOI: $10.1111 / \mathrm{j} \cdot 1600-6143.2011 .03793 . \mathrm{x}$

23. Boon AC, Finkelstein D, Zheng M, Liao G, Allard J, Klumpp K, et al. H5n1 influenza virus pathogenesis in genetically diverse mice is mediated at the level of viral load. mBio. 2011;2(5). doi: 10.1128/mBio.00171-11. Print 2011.
24. Tretyakova I, Pearce MB, Florese R, Tumpey TM, Pushko P. Intranasal vaccination with $\mathrm{h} 5, \mathrm{~h} 7$ and $\mathrm{h} 9$ hemagglutinins co-localized in a viruslike particle protects ferrets from multiple avian influenza viruses. Virology 2013;442(1):67-73. doi: 10.1016/j.virol.2013.03.027. Epub 2013 Apr 22.

25. Kanekiyo M, Wei CJ, Yassine HM, Mc Tamney PM, Boyington JC, Whittle JR, et al. Self-assembling influenza nanoparticle vaccines elicit broadly neutralizing h1n1 antibodies. Nature. 2013; 499(7456):102106. doi: 10.1038/nature12202. Epub 2013 May 22.

26. Trondsen M, Aqrawi LA, Zhou F, Pedersen G, Trieu MC, Zhou P, et al. Induction of local secretory iga and multifunctional cd4(+) t-helper cells following intranasal immunization with a h5n1 whole inactivated influenza virus vaccine in balb/c mice. Scandinavian journal of immunology. 2015;81(5):305-317. doi: 10.1111/sji.12288. 